\title{
Informatik in der Grundschule
}

\section{Eine informatisch-pädagogische Perspektive auf informatikdidaktische Konzepte}

\author{
Ute Schmid · Katharina Weitz • Anja Gärtig-Daugs
}

Received: date / Accepted: date

Zusammenfassung In jüngerer Zeit wendet sich die Informatikdidaktik in Deutschland zunehmend dem Primärbereich zu. Aktuell werden in einem Arbeitskreis der Gesellschaft für Informatik Bildungsstandards für die Grundschule entwickelt und zur Diskussion gestellt. Im vorliegenden Beitrag wird diese Diskussion aufgegriffen. Zunächst werden Konzepte und wissenschaftliche Betrachtungen zur Informatik im Grundschulbereich kurz eingeführt. Danach wird die aktuelle Version der Bildungsstandards vorgestellt und kritisch kommentiert. Die Standards sind zwangsläufig eher allgemein gehalten. Eine Konkretisierun,g was die Anbindung an die kindliche Lebenswelt sowie die Entwicklung von Modulen betrifft, die von Pädagogen im Vor- und Grundschulbereich nachvollzogen und umgesetzt werden können, steht noch aus. Wir stellen ein integratives Konzept für die frühe Informatikbildung in Vor- und Grundschule vor, das von der interdisziplinären Forschungsgruppe Elementarinformatik (FELI) entwickelt wurde. Hier werden einige der in den Bildungsstandards formulierten Kompetenzbereiche addressiert. Dabei werden für die informatischen Konzepte jeweils (a) altersgemäße, 'begreifbare' Materialien zur Verfügung gestellt, (b) Bezüge zwischen den formalen Konzepten und von Kindern genutzten Medien aufgezeigt, und (c) Handreichungen formuliert, die dem Kenntnisstand von pädagogischen Fach- und Lehrkräften angemessen sind.

\section{Ute Schmid}

Kognitive Systeme und

Forschungsgruppe Elementarinformatik

Fakultät Wirtschaftsinformatik und Angewandte Informatik

E-Mail: ute.schmid@uni-bamberg.de

Katharina Weitz

E-Mail: katharina-blandina.weitz@stud.uni-bamberg.de

Anja Gärtig-Daugs

E-Mail: anja.gaertig-daugs@uni-bamberg.de
Schlüsselwörter Informatikunterricht · Grundschule $\cdot$ Kindergarten

\section{Zum aktuellen Stand der Informatikdidaktik für die Grundschule}

Kinder wachsen in einer zunehmend von Informationstechnologien geprägten Welt auf. Informatische Bildung wird deshalb als eine wichtige Kulturtechnik gesehen (Herper und Hinz, 2009), die Kinder zu einem reflektierten Umgang mit digitalen Medien und zur aktiven Beteiligung am Leben in der Informationsgesellschaft und dessen Mitgestaltung befähigen soll (Bundesministerium für Bildung und Forschung, 2016; Kultusminister Konferenz, 2016). Bereits in den Empfehlungen der GI für ein Gesamtkonzept zur informatischen Bildung in allgemeinbildenden Schulen, die im Jahr 2000 formuliert wurden (Breier et al, 2000), setzt informatische Bildung in der Grundschule an: Der Fokus wird dabei auf der Vermittlung von Grundfertigkeiten im Umgang mit Informatiksystemen (Benutzung von Maus und Tastatur, Starten und Beenden von Programmen, Laden, Speichern und Ausdrucken von Dokumenten) und von ersten Grundkenntnissen zu den wichtigsten Systemkomponenten von Informatiksystemen und deren Funktion gesehen. Schwill erkannte bereits 2001, dass die Heranführung von Kindern an die Handhabung eines Computers nicht ohne die Vermittlung von grundlegenden informatischen Konzepten (wie Ordnerstrukturen oder Zweischrittalgorithmen in Form von copy \& paste) erfolgen kann.

Als Grundlage für eine informatische Bildung in der Grundschule, die über die Schulung grundlegender Anwendungskompetenzen hinausgeht, befasste Schwill (2001) sich deshalb mit der Frage, welche fundamentalen Ideen 
der Informatik (wie etwa Rekursion, Greedy-Methode oder strukturierte Zerlegung) bereits Grundschulkindern kognitiv angemessen vermittelbar sind. Weitere wissenschaftliche Arbeiten befassen sich mit der Frage, wie informatische Inhalte in den Grundschulunterricht eingebunden werden können (Borowski et al, 2010), wie im Grundschulalter die Entwicklung algorithmischer Kompetenzen angeregt werden kann (Weigend, 2009; Goecke et al, 2017), wie sich die Fähigkeit zum Algorithmisieren über die Jahrgangsstufen hinweg entwickelt (Hoffmann et al, 2017), welche Programmierkonzepte von Grundschulkindern erlernbar sind (Geldreich et al, 2016) oder inwieweit Robotik-Kits zur frühen Hinführung an informatische Themen geeignet sind (Romeike und Reichert, 2011).

Erste Unterrichtskonzepte und -materialien, mit denen grundlegende informatische Konzepte auch Grundschulkindern näher gebracht werden können, wurden auf internationaler Ebene in den 1990er-Jahren konzipiert und seitdem stetig weiterentwickelt (Bell et al, 1998). Auch in Deutschland wurde seit den 2000er-Jahren eine Vielzahl an Ideen entwickelt. Dabei unterscheiden sich die Konzepte in der Herangehensweise: Teils werden informatische Grundprinzipien und -kompetenzen losgelöst vom Computer (z.B. Gallenbacher, 2007; Borowski et al, 2011; Gallenbacher et al, 2015; Bergner et al, 2011) vermittelt, teils mit Anwendungsbezug am Computer (z.B. Lucke, 2011; Borowski und Diethelm, 2009; Geldreich et al, 2016) ${ }^{1}$, und teils unter Einsatz weiterer Hardware (Borowski, 2013; Best und Thierschmann, 2016; Duwe, 2017) $)^{2}$.

\section{Entwicklung von Bildungsstandards für die Grundschulinformatik}

Mitte September 2017 wurde vom GI-Fachausschuss Informatische Bildung in Schulen ein Dokument mit Empfehlungen zu den Kompetenzen für informatische Bildung im Primarbereich verabschiedet. Dieses soll als Grundlage für die Entwicklung von Bildungsstandards Informatik für den Primarbereich dienen und ist zur Diskussion gestellt. ${ }^{3}$ Dem Dokument liegt die Vision einer informatischen Bildung in der Grundschule zugrunde (S. 4), die prägnant mit den Schlagworten „Ich kann das!“, „Ich entscheide mit!““ und „Hier wird mir geholfen!" beschrieben wird: Informatikunterricht in der Grundschule soll Schülerinnen und Schüler anregen, sich mit Phänomenen aus der Alltagswelt auseinanderzuset-

\footnotetext{
1 siehe auch http://schuelerlabor.informatik. rwth-aachen.de/module/grundschule

2 siehe auch https://calliope.cc/

3 siehe http://metager.to/gibsppdf
}

zen, und sie befähigen, die dahinter liegenden informatisch modellierten Prinzipien zu erkennen, sowie Informatiksysteme kompetent, selbstbestimmt und kritisch reflektiert zu nutzen. Sie werden dabei idealerweise von kompetenten pädagogischen Fach- und Lehrkräften begleitet, die in ihrer Bildungsbiographie selbst zahlreiche Berührungspunkte mit informatischen Themen hatten.

Die Empfehlungen orientieren sich an den Prozessund Inhaltsbereichen, die auch Gegenstand der Bildungsstandards Informatik für die Sekundarstufe I und II sind. Dieser Vorgehensweise liegt das Ziel zugrunde, vom Primarbereich bis zur Sekundarstufe II anschlussfähige Kompetenzerwartungen für die einzelnen Stufen zu formulieren. Zu den Prozessbereichen zählen „Modellieren und Implementieren“, „Begründen und Bewerten“, „Strukturieren und Vernetzen“, „Kommunizieren und Kooperieren" sowie "Darstellen und Interpretieren“. Die Inhaltsbereiche gliedern sich in „Information und Daten“, „Algorithmen", „Sprachen und Automaten“, „Informatiksysteme“ und „Informatik, Mensch und Gesellschaft" (vgl. S. 8). Zu jedem Inhaltsbereich wird in knapper Form der Bezug zur Lebenswelt von Kindern, zum Fach Informatik und den Kompetenzen, die vermittelt werden sollen, verdeutlicht. Eine detaillierte Beschreibung der Kompetenzerwartungen nach Inhaltsbereichen und unter Einbeziehung prozessbezogener Dimensionen schließt sich an. Dabei werden Kompetenzerwartungen jeweils zum Ende der 2. und zum Ende der 4. Klasse formuliert.

Der Vorschlag des GI-Fachausschusses liefert mit den formulierten Kompetenzzielen einen wichtigen ersten Schritt für die didaktische Erschließung des Themas Informatik für den Grundschulbereich. Es fehlen bislang jedoch konkrete methodisch-didaktische Vorschläge zur Ausgestaltung von Unterrichtseinheiten und praktisch erprobte Umsetzungsbeispiele. Für drei ausgewählte Themenbereiche - Digitale Welt, Roboter und Kryptographie - werden derzeit im Bundesland NordrheinWestfalen entwickelt und erprobt. ${ }^{4}$ Das vorgestellte Konzept erhält zwar ein Glossar mit wichtigen informatischen Materialien, dennoch ist davon auszugehen, dass Grundschullehrkräfte, die üblicherweise nur geringe Informatikkenntnisse haben, nicht in der Lage sind, aus den kurz formulierten Hinweisen praktikable Unterrichtskonzepte zu entwickeln, mit denen Unterrichtsstunden gestaltet werden können. Auch die exemplarischen Verdeutlichungen des Lebensweltbezugs sind deutlich $\mathrm{zu}$ knapp, als dass Lehrkräfte darauf aufbauend konkrete Anschauungsbeispiele ableiten können. Es fehlen also bislang praktische Handreichungen und begleitende

\footnotetext{
4 https://www.schulministerium.nrw.de/docs/ Schulsystem/Unterricht/Lernbereiche-und-Faecher/MINT/ Informatik-an-Grundschulen/index.html
} 
Erläuterungen, die auf den Kenntnisstand der Lehrkräfte zugeschnitten sind. Erst wenn durch verständliches Unterrichtsmaterial Barrieren abgebaut werden, besteht die Chance, dass Lehrkräfte, die der Einbindung informatischer Themen gegenüber aufgeschlossen sind und sich an den Empfehlungen zur Informatik für den Primarbereich orientieren möchten, tatsächlich zur Umsetzung im Unterricht motiviert werden.

Betrachtet man die Studienpläne für Lehramt an weiterführenden Schulen im Vergleich zum Grundschullehramt wird deutlich, dass an Grundschulen ein deutlich geringerer Fokus auf Fachwissen gegenüber didaktischen und pädagogischen Aspekten der anschaulichen und motivierenden Vermittlung grundlegender Kompetenzen liegt. Soll das Thema Informatik in die Grundschule integriert werden, ist es entsprechend notwendig, ein geeignetes, didaktisches Konzept zu entwickeln. Dieses sollte nicht ein reines Herunterbrechen der Lehrplaninhalte aus der Sekundarstufe I auf den Grundschulbereich sein. Ähnlich wie bei den frühen naturwissenschaftlichen Inhalten sollte ein spielerischer, forschender Zugang im Vordergrund stehen, der Interesse von Kindern für informatische Themen weckt und ihnen ein Verstehen der digitalisierten Welt ermöglicht.

\section{Eine integrative Perspektive}

Die Forschungsgruppe Elementarinformatik (FELI) an der Universität Bamberg ${ }^{5}$ hat sich im Jahr 2015 gegründet. Dabei kann auf nun bereits zehnjährige praktische Erfahrung mit der Konzeption und Durchführung von Workshops für Kinder verschiedener Altersstufen vom Kindergarten bis zur Oberstufe zurückgegriffen werden (Schmid und Gärtig-Daugs, 2017). Die den Workshops zugrunde liegenden Konzepte wurden und werden überwiegend aus der fachwissenschaftlichen (im Gegensatz zur fachdidaktischen) Perspektive heraus - in Kooperation von Informatik und Pädagogik - gestaltet. Aus der praktischen Erfahrung kristallisierten sich diejenigen didaktischen Konzepte heraus, die sich altersspezifisch besonders bewährt haben - sowohl was die Motivation der Kinder als auch deren kognitiven Voraussetzungen betrifft.

Hinsichtlich der Einführung von Informatik in der Vor- und Grundschule plädieren wir für einen Weg, der verschiedene Perspektiven integriert anstatt $\mathrm{zu}$ spalten, um Kindern und Lehrkräften einen bestmöglichen Einstieg in das spannende Gebiet der Informatik zu ermöglichen. Insbesondere wird unser Ansatz von folgenden Prämissen geleitet:

\footnotetext{
5 siehe www.uni-bamberg.de/kogsys/feli
}

Integration einer medienpädagogischen und einer informatischen Perspektive: Üblicherweise werden diese beiden Bereiche völlig unabhängig voneinander betrachtet. Damit erfahren Kinder auf der einen Seite, wie sie Medien sinnvoll, sicher und reflektiert nutzen können, auf der anderen Seite, erlernen sie informatische Konzepte, etwa Datenstrukturen und Algorithmen. Wir gehen davon aus, dass der häufig als ,zweites Mathe-Fach" erlebte Informatikunterricht durch den vielfältigen Einsatz von Computermedien motivierender gestaltet werden kann. Umgekehrt hilft die Vermittlung von Informatikkonzepten im Zusammenhang mit der Vermittlung von Mediennutzung zu einem vertiefteren und ganzheitlichen Verständnis von Computermedien. Erst die Kombination beider Bereiche, insbesondere für die Vor- und Grundschule, kann Kinder anregen, Wiefunktioniert-das Fragen zu stellen und statt bloßem Handlungswissen transferierbares Konzeptwissen aufzubauen.

Integration von unplugged Ansätzen, Programmierung und Computeranwendung: Traditionell wird im Informatikunterricht (ab der Sekundarstufe) die Vermittlung logisch-algorithmischer Grundlagen in den Mittelpunkt gestellt. Auch einige Ansätze für Kindergarten (Schwill, 2001) und Grundschule (Bell et al, 1998) folgen diesem Vorgehen. Aktuell wird dagegen für den Grundschulbereich direkt auf die Programmierung - etwa mit Scratch (Resnick et al, 2009; Fessakis et al, 2013), Calliope mini (Calliope, 2017) ${ }^{6}$ oder Roberta (Jost et al, 2014) gesetzt. Computeranwendung, etwa die Nutzung von Malprogrammen wie TuxPaint, oder Internetrecherche wird dagegen häufig der Medienpädagogik überlassen. Unserer Meinung nach kann ein direkter Einstieg in die Programmierung nach dem Vorbild der frühen Arbeiten von Papert (1980) als entdeckendes Lernen durchaus sinnvoll sein, um Kinder spielerisch und bottom-up an zentrale Konzepte wie Variablen, bedingte Anweisungen und Schleifen heranzuführen. Dennoch ist davon auszugehen, dass bei der Altersgruppe der bis 10-jährigen auf unmittelbar begreifbare Materialien im Sinne der Montessori Pädagogik unverzichtbar sind, damit es gelingt konzeptuelles Wissen aufzubauen. Zudem kann auch die Nutzung von Computeranwendungen als motivierender Ausgangspunkt für die Einführung von informatischen Konzepten dienen. So kann die Nutzung eines Malprogramms die Konzepte Pixel und digitale Repräsentation konkret werden lassen.

Parallele Entwicklung von Unterrichtsmaterialien und Handreichungen für Lehrpersonen: Häufig wird die Forderung nach digitaler Bildung dadurch umgesetzt, dass Schulen mit neuer Hardware und

\footnotetext{
6 siehe auch https://calliope.cc
} 


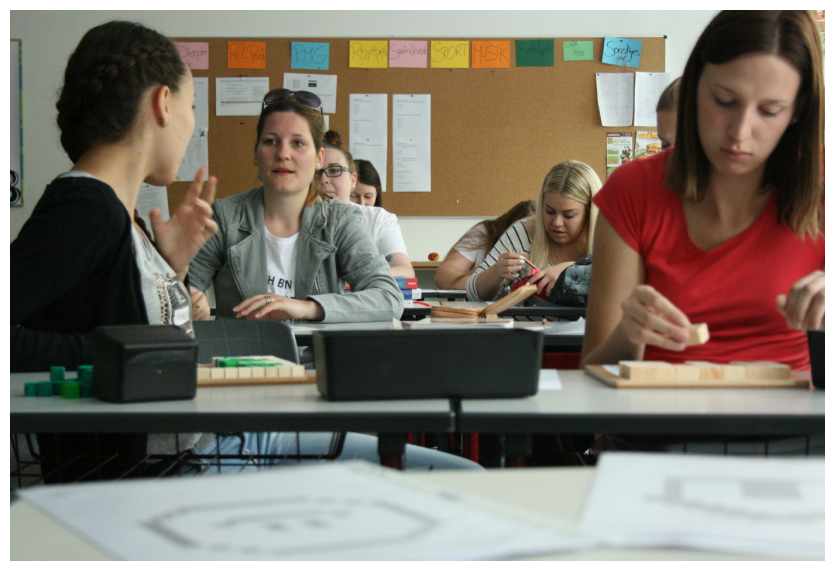

Abb. 1 Fachkräfte mit ins Boot holen: Einbezug pädagogischer Fachkräfte bei der Entwicklung kindgerechter Materialien für die Experimentierkiste.

Infrastruktur ausgestattet werden. Dabei wird übersehen, dass Lehrkräfte ohne Konzepte, wie die Computer sinnvoll im Unterricht eingesetzt werden können, sowie ohne Handreichungen, die für ihren eigenen Kenntnisstand angemessen sind, kaum in der Lage sind, Informatikkompetenzen zu vermitteln. Insbesondere im Grundschulbereich haben viele Lehrkräfte keinerlei informatische Vorkenntnisse und sind zudem häufig technikängstlich. Nur wenn konkrete Unterrichtseinheiten ausgearbeitet vorliegen, die zudem Schritt-für-Schritt nachvollziehbar beschrieben sind, werden Lehrkräfte die vorhandene Hardware auch nutzen. Mittelfristig werden entsprechende Kenntnisse Eingang in die Lehramtsausbildung und die Erzieherausbildung finden. Aber auch hier werden - nach dem Vorbild des Sachunterrichts - spezifische Ansätze notwendig sein, die sich deutlich von der fachspezifischen Ausbildung von Lehrkräften des Gymnasiums unterscheiden müssen (siehe Abb. 1, Weitz et al, 2017).

Informatik als Querschnittsdisziplin statt als weiteres Unterrichtsfach: Derzeit wird immer wieder gefordert, Informatik als weiteres Unterrichtsfach in der Grundschule einzuführen. Damit werden die ohnehin bereits überfrachteten Lehrpläne weiter aufbeläht oder es müssen andere Fächer im Stundenumfang gekürzt werden. Dies scheint uns nicht zielführend und wird auch von vielen Eltern abgelehnt, die zurecht fordern, dass die Kinder zunächst die Basiskompetenzen des Lesens, Schreibens und Rechnens erlernen sollen. Wir plädieren dafür, Informatik als Brückenfach in verschiedene Unterrichtsfächer zu integrieren. Dabei ist eine Auseinandersetzung mit Informatikthemen im Umfang von bis zu 10 Stunden pro Schuljahr denkbar. Konzepte wie digitale Repräsentation können beispielsweise im Zusammenhang mit Pixeln als Projekt digitales Gestalten im Kunstunterricht vermittelt werden. Algo-

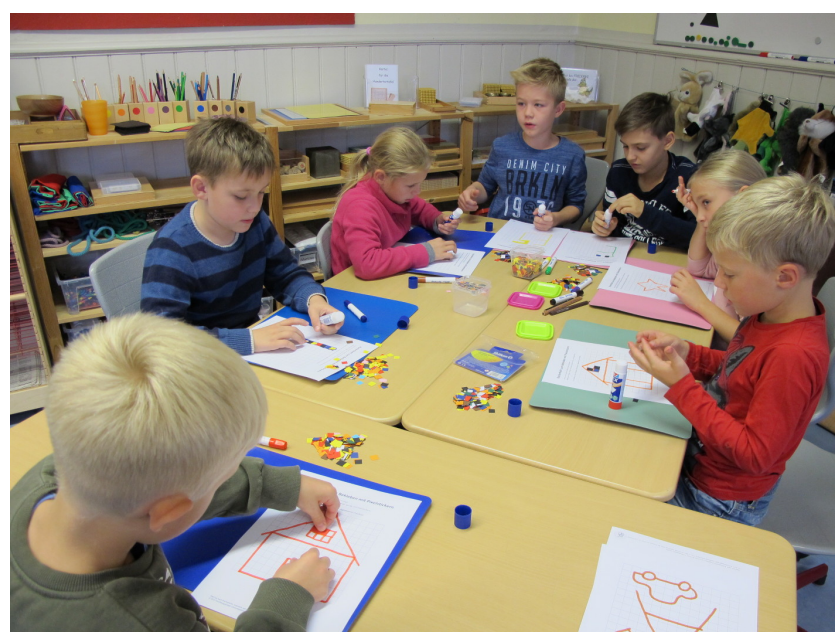

Abb. 2 Empirische Evaluation der Materialien in einer Grundschule.

rithmen finden sich im Mathematikunterricht - etwa bei der Einführung der schriftliche Subtraktion, aber auch im Deutschunterricht beim Thema Vorgangsbeschreibungen (Schmid und Gärtig-Daugs, 2017).

Praktischen Maßnahmen fachlich und pädagogisch begründet und empirische evaluiert: Die konkrete Ausgestaltung von praktisch umsetzbaren Unterrichtseinheiten sollte im Mittelpunkt jeder fachdidaktischen Bemühung stehen. Natürlich müssen die Inhalte der Lernmodule auf fachlich-inhaltlichen Überlegungen begründet sein. So liegt es nahe, dass Algorithmen als zentrales Konzept der Informatik sich in Lerneinheiten für jede Altersstufe finden sollten. Zudem sollten aber insbesondere für Kinder bis zehn Jahre nicht nur didaktische sondern auch pädagogische und lernpsychologische Ansätze für die konkrete Ausgestaltung einer altersgerechten Vermittlung von Inhalten herangezogen werden. Es ist eine Selbstverständlichkeit, dass die Wirkung der entwickelten Konzepte empirisch überprüft werden muss. Dabei sind sowohl qualitative, auf geringe Fallzahlen begrenzte Studien als auch breiter angelegte Fragebogenstudien sinnvoll. Eine Erhebung, wie gut die vermittelten Konzepte erworben wurden, sollte gerade in der Grundschule wenn möglich nicht in Form von Leistungstests erfolgen. Alternativ können Ratespiele und Transferaufgaben gestellt werden. Daneben sollte immer auch geprüft werden, wie motivierend die Materialien sind. Ohne Motivation wird sich kein Kind vertiefend, auch außerhalb der Schule, weiter mit Informatikthemen, beispielsweise mit der Programmierung eigener Projekte, befassen. Ohne Motivation wird Informatik auch nicht als späterer Berufswunsch in Betracht gezogen werden (siehe Abb. 2, Wolking und Schmid, 2017). 
Spiralcurriculum vom Kindergarten bis zur Oberstufe: Wie bei der Erschließung jedes Fachs, ist es auch für die Vermittlung informatischer Kompetenzen wichtig, dass Inhalte in verschiedenen Phasen der kognitiven Entwicklung wiederholt aufgegriffen und jeweils altersangemessen vertieft werden. Beispielsweise kann ein grundlegendes und anschauliches Konzept wie Pixel durchaus in der Sekundarstufe wieder als Einstieg in das Thema Binäres Zahlensystem genutzt werden. Ein einfacher, in der dritten Klasse erarbeiteter Sortieralgorithmus wie selection sort kann später systematischer betrachtet und mit effizienteren Algorithmen wie quicksort verglichen werden (Bell et al, 1998).

Interdisziplinäre Perspektive auf Materialentwicklung: Während die Fachdidaktik im Unterricht für die Mittel- und Oberstufe durchaus überzeugende Konzepte entwickelt hat, scheint uns die von dieser Disziplin eingenommene Perspektive für den Elementar- und Primärbereich nur bedingt hilfreich. Für jüngere Kinder, die in der kognitiven Entwicklung noch weit vor der formalen Phase (Piaget) liegen, darf nicht allein das fachliche Thema im Mittelpunkt stehen. Hier liefern die Pädagogik sowie die kognitiven Lerntheorien einschlägige Konzepte zur kindgerechten Vermittlung abstrakter Inhalte. Hierzu gehören die bereits genannten Konzepte der Montessori-Pädagogik. Aber auch Ansätze wie das Nutzen von analogem Schließen zur Förderung des Aufbaus sinnvol generalisierter Wissenstrukturen sind in diesem Kontext relevant (Zeller und Schmid, 2016; Gentner et al, 2016). Während es in der Mittelstufe genügen kann, ein Konzept anhand eines einzelnen Beispiels einzuführen, sind jüngere Kinder darauf angewiesen, die Gemeinsamkeit in einer Vielfalt von Anwendungsbeispielen zu entdecken, um sich vom Einzelfall zu lösen und transferierbares und beständiges Wissen aufzubauen. So kann etwa das Konzept Pixel erarbeitet werden, in dem Pixelbilder geklebt werden, ein gemaltes Bild in Pixel überführt wird, und Bilder mit grober Aufösung immer feiner gezoomt werden.

\section{Illustration des Konzepts am Beispiel Pixel}

Das beschriebene Konzept haben wir in einer Experimentierkiste Informatik umgesetzt, in der Materialien zu verschiedenen Themen zusammengestellt sind. Begleitend zur Kiste existiert eine umfangreiche Handreichung für die Lehrkräfte, in der alle Lernmodule konkret beschrieben sind.

Als Einstiegsthema, das bereits im Kindergarten behandelt werden kann, dient in der Experimentierkiste das Thema Pixel. Das Thema Pixel ermöglicht einerseits die Einführung erster Informatikkonzepte und schlägt andererseits die Brücke zwischen der Erfahrungswelt

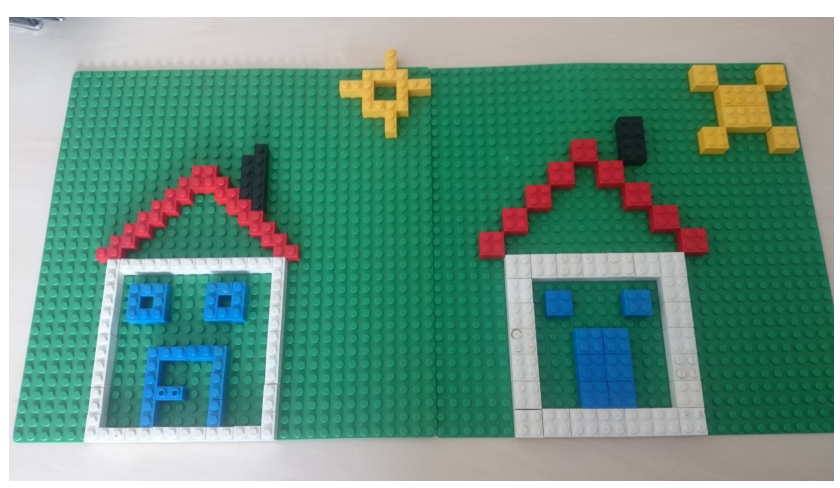

Abb. 3 Legohaus zur Veranschaulichung des Konzepts Pixel in der von FELI entwickelten Experimentierkiste Informatik.

der Kinder und neuen Wissensbereichen. Ausgehend von Alltagserlebnissen und mit bekannten Materialien (wie Lego, Stifte, Holzbausteine) wird der Einstieg in das Thema gestaltet. Dies ermöglicht es, die Kinder in ihrer Lebenswelt abzuholen. Zunächst werden bei dem Thema Pixel mit Buntstiften gemalte Bilder mit am Computer gemalten Bildern verglichen. Unterschiede und Gemeinsamkeiten werden erarbeitet. Dann wird zu der Leitfrage für den Themenbereich Pixel übergeleitet: Wie stellt ein Computer Bilder dar? Eine erste Veranschaulichung des Konzeptes Pixel geschieht mithilfe von Legosteinen, die auf einer Legoplatte zu einem Motiv (zum Beispiel einem Haus) angeordnet sind (siehe Abb. 3). Dieses Motiv wird einmal mit kleinen und einmal mit großen Legosteinen gestaltet. Jeder Legostein stellt hierbei ein Pixel dar. Durch den Vergleich der beiden Motive wird deutlich, dass die Größe der Pixel Einfluss auf die Darstellung hat. Je kleiner die Pixel (Legosteine) sind, desto detaillreicher ist das Bild.

Der Bezug zum Computer wird hergestellt, indem eine - eventuell von den Kindern gemachte - Digitalfotografie in verschiedenen Auflösungen auf dem Bildschirm angezeigt wird. Die Kinder können entdecken, dass die Bilder, die sie normalerweise hochaufgelöst sehen, tatsächlich aus Pixeln aufgebaut sind. Sehr motivierend ist hier immer, wenn das Ansehen der Bilder als Ratespiel in Schritten von geringer, sehr verpixelter, zu hoher Auflösung gestaltet wird.

In einem nächsten Schritt kann das vorgestellte Konzept durch verschiedene Experimentiermaterialien von den Kindern selbst erfahren werden. Die Experimentiermaterialien sind dabei anlehnt an die Ideen Maria Montessoris, Materialien zur Verfügung zu stellen, die Kindern ein „be-greifen“ ihrer Welt ermöglichen. So können auf „Pixelbildern“ vergleichbar zu dem Konzept „Malen nach Zahlen“ Motive ausgemalt werden. Hierfür müssen die Kinder quadratische Kästchen, die mit einer 0 beschriftet sind, ausmalen, während Kästchen, die mit einer 1 beschriftet sind, nicht ausgemalt wer- 


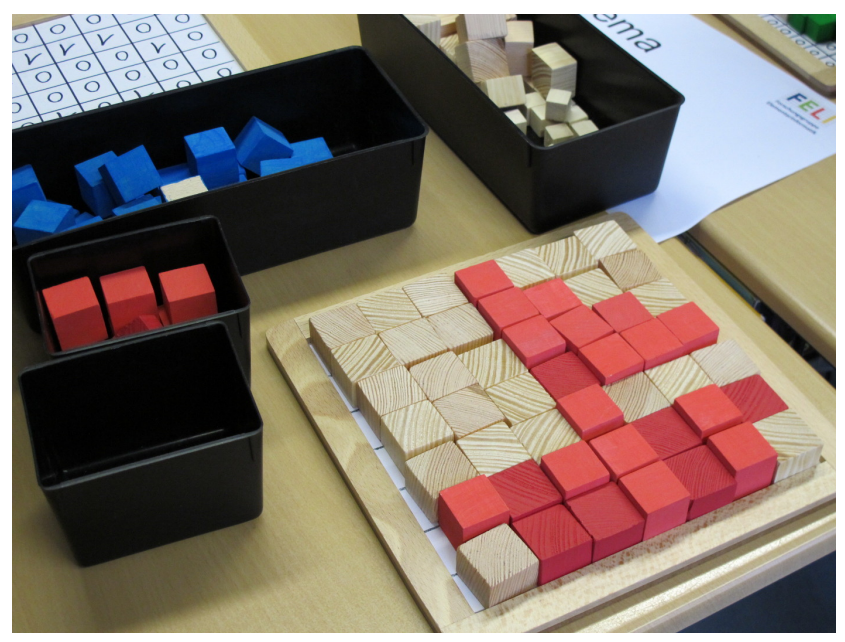

Abb. 4 Setzspiel mit Holzwürfeln zur Veranschaulichung des Konzepts Pixel, entwickelt von FELI.

den. Durch dieses Vorgehen erhält man ein sogenanntes Binärbild. Bei einem anderen Experimentiermaterial legen die Kinder mithilfe von bemalten und unbemalten Holzwürfeln ein Binärbild in einem Holzrahmen, der mit einem Blatt auf dem Kästchen mit Nullen und Einsen aufgezeichnet sind, ausgelegt ist (siehe Abb. 4). Hier gibt es, ähnlich wie bei dem vorgestellten Legosteinen am Anfang, kleine und große Holzwürfel. Die Erstellung eines Binärbildes mit den kleinen Holzwürfeln dauert deutlich länger als die Erstellung eines Bildes mit großen Holzwürfeln. Dafür ist der Detaillierungsgrad deutlich höher. Sogenanntes „Expertenmaterial“ fordert Kinder, die Binärbilder mit eigenen Motiven gestalten wollen. Sie können den unbeschrifteten Kästchen auf einem Blatt selbst Nullen und Einsen zuordnen und so ihr eigenes Binärbild entwickeln.

In einer zusätzlichen Unterrichtsstunde können die Kinder mit einem Malprogramm wie Tuxpaint ${ }^{7}$ selbst ein Bild am Computer gestalten. Auch dieses selbstgemalte Bild kann danach in verschiedenen Auflösungen betrachtet werden.

Die Erläuterungen zum Konzept Pixel sowie Hintergrundinformationen für pädagogische Fachkräfte sind in der der Experimentierkiste beigefügten Handreichung zu entnehmen. Dabei wird darauf geachtet, dass die Umsetzung im Unterricht mit wenig Vorarbeit möglich ist. Die Kompetenzziele, die Anbindung an den Lehrplan, die Einordung in ein Unterrichtsfach, die benötigte Zeit, sowie alle benötigten Materialien werden übersichlich angegeben. Technische Aspekte wie etwa das Präsentieren von digitalen Bildern mit verschiedenen Auflösungen werden Schritt für Schritt beschrieben. Gezielte Hintergrundinformationen und Hinweise sollten

7 http://tuxpaint.org/ helfen, dass Fehlkonzepte wie 'Pixel sind Bausteine' oder 'Pixel sind nur schwarz/weiß' vermieden werden.

Aufbauend auf das Thema Pixel können weiterführende Themen eingeführt werden, etwa Analoge und $D i$ gitale Repräsentationen mit dem Fokus auf der Transformation von analogen in digitale Darstellungen und Computer, bei dem die Anwendung des Konzeptes im Kontext von Computer und Tablet behandelt wird. Das Thema Pixel ist schwerpunktmäßig für den Vor- und Grundschulbereich konzipiert worden, kann aber in der Sekundarstufe nahtlos weitergeführt werden. So kann die Erstellung von Binärbildern eine Grundlage für das Themengebiet Binäres Zahlensystem liefern.

\section{Fazit}

Nach einer zweijährigen Erprobung der Experimentierkiste Informatik in zahlreichen Kindertagesstätten und Grundschulen und deren kontinuierliche Weiterentwicklung freuen wir uns, dass die Kiste bei vielen pädagogischen Fach- und Lehrkräften sehr großen Anklang findet und über mehrere Monate voraus ausgebucht ist. Unsere Gruppe hat sich aus der Informatik heraus entwickelt als die Initiatorin erst vom Kindergarten ihrer Tochter und später von der Grundschule ihrer Tochter angefragt wurde, ob sie den Kindern nicht 'mal was über Informatik' erzählen könnte. Ähnliche Impulse berichten auch andere Akteure im Bereich der Informatik für jüngere Kinder, allen voran die Autoren von Computer Science Unplugged (Bell et al, 1998).

Dass unser Konzept nicht aus der Informatikdidaktik heraus entwickelt wurde, mag bedingen, dass wir - zumindest zu Beginn unseres Projekts - einschlägige Arbeiten aus diesem Bereich nicht zur Kenntnis genommen haben. Andererseits haben wir recht früh Wissenschaftlerinnen und Wissenschaftler aus den Bereichen Elementarpädagogik, Grundschuldidaktik und Kognitionspsychologie in unser Team integriert und Kontakte zur Medienpädagogik, zu Erzieherinnen und Erziehern sowie Lehrkräften der Grundschule hergestellt. Wir sind fest davon überzeugt, dass diese integrative Perspektive sehr hilfreich ist, um den Schritt von der Forschung hinein in die Praxis der frühkindlichen Informatikbildung zu schaffen.

Danksagung Die Forschungsgruppe FELI wird von der Joachim Herz Stiftung, von der Hermann Gutmann Stiftung und von der Technologie Allianz Oberfranken (TAO) finanziell unterstützt. Wir bedanken uns herzlich für die Förderungen ohne die unsere Arbeit nicht möglich wäre. Sehr herzlich bedanken wir uns auch bei Damaris Güting vom Landesinstitut für Lehrerbildung und Schulentwicklung Hamburg, die uns mit ihrem Enthusiasmus für unsere Experimentierkiste immer wieder motiviert, auch gegen Widrigkeiten weiterzuma- 
chen. Nicht zuletzt danken wir allen Grundschullehrerinnen und -lehrern, Erzieherinnen und Erziehern, die unsere Experimentierkiste teilweise schon mehrfach ausgeliehen haben und uns so viel nützliche und motivierende Rückmeldung geben, allen voran Carina Neubauer.

\section{Literatur}

Bell TC, Witten IH, Fellows M (1998) Computer Science Unplugged: Off-line activities and games for all ages. Computer Science Unplugged

Bergner N, Leonhardt T, Schroeder U (2011) Zauberschule Informatik-Einblick in die Welt der Informatik für Kinder im Grundschulalter. In: Weigend M, Thomas M, Otte F (eds) Informatik mit Kopf, Herz und Hand, ZfL-Verlag, Münster, pp 132-141

Best A, Thierschmann U (2016) Erste Erfahrungen beim Einsatz von Unterrichtsbausteinen zur kooperativen Entwicklung und Erprobung von Informatikstunden an Grundschulen. In: Informatik 2016, Gesellschaft für Informatik

Borowski C (2013) Kinder auf dem Weg zur Informatik: Roboter in der Grundschule. In: INFOS (GIFachtagung Informatik und Schule), pp 21-28

Borowski C, Diethelm I (2009) Kinder auf dem Wege zur Informatik: Programmieren in der Grundschule. In: INFOS (GI-Fachtagung Informatik und Schule), vol 13

Borowski C, Diethelm I, Mesaroş AM (2010) Informatische Bildung im Sachunterricht der Grundschule. wwwwiderstreit-sachunterrichtde 15

Borowski C, Dehé M, Hühnlein F, Diethelm I, Ossietzky Cv (2011) Kinder auf dem Weg zur Informatik: Wie funktioniert das Internet. In: Weigend M, Thomas M, Otte F (eds) Informatik mit Kopf, Herz und Hand, ZfL-Verlag, Münster

Breier N, Fothe M, Friedrich S, Hubwieser P, Koerber B, Röhner G, Schubert S, Seiffert M (2000) Empfehlungen für ein Gesamtkonzept zur informatischen Bildung an allgemein bildenden Schulen. Tech. rep., Gesellschaft für Informatik eV (GI)

Bundesministerium für Bildung und Forschung (2016) Bildungsoffensive für die digitale Wissensgesellschaft: Strategie des Bundesministeriums für Bildung und Forschung. URL https://www.bmbf .de/pub/Bildungsoffensive_ fuer_die_digitale_Wissensgesellschaft.pdf

Calliope (2017) Coden mit dem Calliope mini - Programmieren in der Grundschule. Lehrermaterial für den Einsatz ab Klasse 3. Cornelsen

Duwe W (2017) Die Handykiste für die Grundschule. In: INFOS - Informatische Bildung zum Verstehen und Gestalten der digitalen Welt, pp 413-414
Fessakis G, Gouli E, Mavroudi E (2013) Problem solving by $5-6$ years old kindergarten children in a computer programming environment: A case study. Computers \& Education 63:87-97

Gallenbacher J (2007) Abenteuer Informatik: IT zum Anfassen; von Routenplaner bis Online-Banking. Elsevier

Gallenbacher J, Gose K, Heun D (2015) Gestrandet auf der Schatzinsel - Schätze heben mit Informatik in der Grundschule. In: INFOS (GI-Fachtagung Informatik und Schule), pp 101-109

Geldreich K, Funke A, Hubwieser P (2016) A programming circus for primary schools. ISSEP 2016 p 49

Gentner D, Levine SC, Ping R, Isaia A, Dhillon S, Bradley C, Honke G (2016) Rapid learning in a children's museum via analogical comparison. Cognitive science 40(1):224-240

Goecke L, Stiller J, Pech D, Pinkwart N (2017) Informatische Grundbildung: Exploration des Erstzugangs zu Lego® Wedo 2.0 und Cubelets von Drittklässler_innen. In: INFOS - Informatische Bildung zum Verstehen und Gestalten der digitalen Welt, Gesellschaft für Informatik, Bonn

Herper H, Hinz V (2009) Informatische Bildung im Primarbereich. In: INFOS (GI-Fachtagung Informatik und Schule), pp 74-85

Hoffmann S, Wendlandt K, Wendlandt M (2017) Algorithmisieren im Grundschulalter. In: INFOS - Informatische Bildung zum Verstehen und Gestalten der digitalen Welt, Gesellschaft für Informatik, Bonn

Jost B, Ketterl M, Budde R, Leimbach T (2014) Graphical programming environments for educational robots: Open roberta-yet another one? In: Multimedia (ISM), 2014 IEEE International Symposium on, IEEE, pp 381-386

Kultusminister Konferenz (2016) Bildung in der digitalen Welt: Strategie der Kultusministerkonferenz. URL https://www . kmk.org/aktuelles/artikelansicht/ strategie-bildung-in-der-digitalen-welt. html

Lucke U (2011) Das Computer-Freundebuch: Ein Ansatz für Informatik in der Grundschule. In: Weigend M, Thomas M, Otte F (eds) Informatik mit Kopf, Herz und Hand, ZfL-Verlag, Münster, pp 207-214

Papert S (1980) Mindstorms: Children, computers, and powerful ideas. Basic Books

Resnick M, Maloney J, Monroy-Hernández A, Rusk N, Eastmond E, Brennan K, Millner A, Rosenbaum E, Silver J, Silverman B, et al (2009) Scratch: programming for all. Communications of the ACM 52(11):6067 
Romeike R, Reichert D (2011) PicoCrickets als Zugang zur Informatik in der Grundschule. In: INFOS (GIFachtagung Informatik und Schule), pp 177-186

Schmid U, Gärtig-Daugs A (2017) Zehn Jahre Elementarinformatik - Elementare Informatikkompetenzen als Basis für nicht-konsumierenden und reflektierten Umgang mit Computer-Medien in der Vor- und Grundschule, 2008-2017. Tech. Rep. Band 1, Werkstattbericht zur Elementarinformatik, Forschungsgruppe FELI, Universität Bamberg, URL https://opus4.kobv.de/opus4-bamberg/ frontdoor/index/index/docId/49769

Schwill A (2001) Ab wann kann man mit Kindern Informatik machen? In: INFOS (GI-Fachtagung Informatik und Schule), pp 13-30

Weigend M (2009) Algorithmik in der Grundschule. In: INFOS (GI-Fachtagung Informatik und Schule), pp 97-108

Weitz K, Gärtig-Daugs A, Knauf D, Schmid U (2017) Computer science in early childhood education: Pedagogical beliefs and perceived self-confidence in preschool teachers. In: Barendsen E, Hubwieser P (eds) Proceedings of the 12th Workshop on Primary and Secondary Computing Education (WiPSCE 2017, Nijmegen, The Netherlands, November 08 - 10), ACM, pp 117-118

Wolking M, Schmid U (2017) Mental models, career aspirations, and the acquirement of basic concepts of computer science in elementary education: Empirical evaluation of the computer science experimenter's kit. In: Barendsen E, Hubwieser P (eds) Proceedings of the 12th Workshop on Primary and Secondary Computing Education, (WiPSCE 2017, Nijmegen, The Netherlands, November 08 - 10), ACM, pp 119 120

Zeller C, Schmid U (2016) Automatic generation of analogous problems for written subtraction. In: Proceedings of the 14th International Conference on Cognitive Modeling (ICCM 2016), pp 241-242 